\title{
Del esplendor porfirista al caos revolucionario. La enseñanza primaria en el Estado de Chihuahua, México, en el período 1892 a 1911*
}

\author{
Jesús Adolfo Trujillo Holguín
}

Profesor de la Universidad Autónoma de Chihuahua (México).Correo electrónico: jatrujillo@uach.mx. El autor es doctor en Educación de la Universidad Autónoma de Chihuahua (México). Entre sus publicaciones recientes tenemos: "Alcances y limitaciones de los archivos históricos en la formación de investigadores. El caso de la Licenciatura en Historia de la Universidad Autónoma de Chihuahua", en Acercamientos a la historia de la educación. Diálogos, actores y fuentes en la construcción del conocimiento histórico, Guillermo Hernández Orozco, Francisco Alberto Pérez Piñón y Jesús Adolfo Trujillo Holguín (México: Nautilium, 2017). Entre sus temas de interés están historia e historiografía de la educación y formación de maestros en Chihuahua, México.

\section{Francisco Alberto Pérez Piñón}

Profesor de la Universidad Autónoma de Chihuahua (México). Correo electrónico: aperezp@uach.mx. El autor es doctor en Ciencias de la Educación del Instituto Superior Pedagógico "José Enrique Varona" (Cuba). Entre sus publicaciones recientes tenemos: "La Hacienda de Coyotillos, un acercamiento a los contenidos educativos", en Acercamientos a la historia de la educación. Diálogos, actores y fuentes en la construcción del conocimiento histórico, Guillermo Hernández Orozco, Francisco Alberto Pérez Piñón y Jesús Adolfo Trujillo Holguín (México: Nautilium, 2017). Entre sus temas de interés están historia e historiografía de la educación.

\section{Guillermo Hernández Orozco}

Profesor de la Universidad Autónoma de Chihuahua (México). Correo electrónico: ghernand@uach.mx. El autor es doctor en Ciencias de la Educación del Instituto Superior Pedagógico "José Enrique Varona" (Cuba). Entre sus publicaciones recientes tenemos: "José Joaquín Calvo López, fundador del Instituto Literario, hoy Universidad Autónoma de Chihuahua", en IE Revista de Investigación Educativa de la Rediech, Vol. 8, No. 14, (2017). Entre sus temas de investigación podemos contar los referentes a Historia e historiografía de la educación en Chihuahua, México.

Recibido: 4 de abril de 2016

Aprobado: 17 de mayo de 2016

Modificado: 30 de mayo de 2016

Artículo de investigación científica

DOI: http://dx.doi.org/10.15648/hc.32.2018.6

* Este artículo forma parte del proyecto titulado: "Historia de la Educación en Chihuahua" financiado por el Programa para el Desarrollo Profesional Docente - PRODEP (México).

Esta publicación está bajo una licencia Creative Commons Reconocimiento-NoComercial 4.0 
Del esplendor porfirista al caos revolucionario. La enseñanza primaria en el estado de Chihuahua, México, en el período 1892 a 1911

\title{
Resumen
}

Este artículo analiza el desarrollo de la enseñanza primaria en el Estado norteño de Chihuahua, México, durante el período de esplendor de la dictadura del general Porfirio Díaz y su transición al proyecto educativo revolucionario (1892-1911). Para tal cometido, se escudriñaron tres archivos: Archivo de Concentración de Gobierno del Estado de Chihuahua (ACGECH) y los Archivos Históricos de la Universidad Autónoma de Chihuahua (AUACH) y del Congreso del Estado (AHCECH), y se consultó bibliografía secundaria. Busca aportar elementos para la revisión de un tema que ha sido estigmatizado en el discurso historiográfico, a pesar de que el Porfiriato contribuyó a la consolidación del sistema educativo estatal. Enfatiza en dos administraciones gubernamentales que le apostaron a la educación como medio para que las personas enfrentaran las demandas sociales del momento.

Palabras clave: porfiriato, historia de la educación, enseñanza primaria, educación en Chihuahua, educación básica.

From the Porfirio splendor to the revolutionary chaos. Primary education in the state of Chihuahua, Mexico, in the period 1892 to 1911

\begin{abstract}
This article discusses the development of primary education in the norther state of Chihuahua, Mexico, during the brightest period of Porfirio Diaz dictatorship and its shift to the revolutionary school project (1892-1911). For such purpose, three archives were analyzed: The Archive of Government Concentration of Chihuahua State (ACGECH) and the Historical Archives of Universidad Autónoma of Chihuahua (AUACH) and the Congress of the State (AHCECH) as well as secondary sources. This paper also seeks to provide elements for the review of an issue that has been stigmatized in the historiographic debate, in spite of the fact that Porfirio contributed to the state education system consolidation. It also emphasizes in two government administrations that put effort to education as a mean for people to face the social demands of the moment.
\end{abstract}

Key words: porfiriato, history of education, primary education, education in Chihuahua, basic education 
Do esplendor porfirista ao caos revolucionário. O ensino primário no estado de Chihuahua, México, no período 1892 a 1911

\section{Resumo}

Este artigo analisa o desenvolvimento do ensino primário no estado norteño de Chihuahua, México, durante o período de esplendor da ditadura do general Porfirio Díaz e sua transição ao projecto educativo revolucionário (1892-1911). Para tal cometido, se escudriñaron três arquivos: Arquivo de Concentração de Governo do Estado de Chihuahua (ACGECH) e os Arquivos Históricos da Universidade Autónoma de Chihuahua (AUACH) e do Congresso do Estado (AHCECH), e consultou-se bibliografia secundária. Procura contribuir elementos para a revisão de um tema que tem sido estigmatizado no discurso historiográfico, apesar de que o Porfiriato contribuiu à consolidação do sistema educativo estatal. Enfatiza em duas administrações governamentais que lhe apostaram à educação como médio para que as pessoas enfrentassem as demandas sociais do momento.

Palavras-chave: porfiriato, história da educação, ensino primário, educação em Chihuahua, educação básica.

\section{De la splendeur « porfirista » au chaos révolutionnaire. L'enseignement primaire dans l'État de Chihuahua, au Mexique, entre 1892 et 1911}

\section{Résumé}

On analyse le développement de l'enseignement primaire dans l'État de Chihuahua, situé au nord du Mexique, pendant la période de la splendeur de la dictature du général Porfirio Díaz et sa transition au projet éducatif révolutionnaire (1892-1911). Dans ce but, on a fouillé trois archives: Archive de Concentration du Gouvernement de l'État de Chihuahua (ACGECH) et les Archives Historiques de l'Universidad Autónoma de Chihuahua (AUACH) et du Congrès de l'État (AHCECH) et l'on a consulté des sources secondaires. Ce travail vise à apporter des éléments pour la révision d'un sujet qui a été stigmatisé dans le discours historiographique, bien que le «Porfiriato» ait contribué à la consolidation du système éducatif national. On met l'accent sur deux administrations gouvernementales qui ont misé sur l'éducation comme le moyen pour que les personnes affrontent les demandes sociales de ce moment-là.

Mots clés: «porfiriato», histoire de l'éducation, enseignement primaire, éducation dans l'État de Chihuahua, éducation basique. 


\section{INTRODUCCIÓN}

En México se conoce con el nombre de Porfiriato al período comprendido entre los años 1876 a 1911, donde estuvo como figura central de la escena política nacional el general Porfirio Díaz Mori ${ }^{1}$, y como Revolución Mexicana a la lucha armada que inicia oficialmente el 20 de noviembre de 1910 para acabar con el régimen. De esta manera, la historia oficial contemporánea ha promovido una visión social en donde el primer período representa el oscurantismo y la causa de las grandes injusticias nacionales, en tanto que lo segundo se considera como la base sobre la cual se edificó la moderna institucionalidad del país.

El ámbito educativo obedece a la misma tendencia. La historiografía tradicional explica el desarrollo y expansión del sistema educativo nacional como fenómeno derivado del movimiento revolucionario, a partir del cual se gestó el ideario de educación popular plasmado en la Constitución Política de 1917 y consolidado con el surgimiento de la Secretaría de Educación Pública (SEP) en 1921. La génesis del sistema educativo con carácter unificador y nacionalista se expone como resultado de la obra educativa de José Vasconcelos, del período de expansión acelerada de la educación rural de la década de 1920, de la federalización educativa y de la reorganización de los tipos y modalidades educacionales.

En el recuento de los avances del período posrevolucionario, incluso se ha denostado el período porfirista como etapa de escasos logros y germen de la inequidad social que se resolvió finalmente al triunfo del movimiento armado. Las investigaciones más serias presentan como balance del gobierno de Porfirio Díaz un panorama educativo desalentador en donde reinaba -además de las profundas desigualdades sociales- "escuelas pri-

$1 \quad$ El general Porfirio Díaz ocupó la presidencia de la República en el primer período constitucional de 1877 a 1880 y el segundo correspondiente a la dictadura de 1884 a 1911. Ejerció el poder de facto desde 1876 hasta su renuncia al cargo el 25 de mayo de 1911, ver más en Pablo Serrano Álvarez, Porfirio Díazy el Porfiriato. Cronología 1830-1915 (México: Instituto Nacional de Estudios Históricos de las Revoluciones de México, 2012), 42-67. 
marias en condiciones lamentables y colegios que más bien parecían conventos medievales" .

Quizás un elemento que ha favorecido la generalización de los acontecimientos históricos es la centralización de las actividades investigativas. En los esfuerzos realizados por grupos académicos como el Consejo Mexicano de Investigación Educativa (COMIE), se ha estudiado la producción en el área de la historia e historiografía de la educación -agrupada por décadas- encontrando una tendencia hacia "la indagación en historias regionales y locales, que ha permitido a su vez repensar los grandes hitos de la historia nacional"'3, en parte debido a la diversificación en el uso de fuentes para construir el relato histórico.

Los fenómenos históricos en Chihuahua tuvieron su propia dinámica y distan mucho de poder explicarse bajo las mismas variables. Algunas investigaciones locales han renunciado a la tendencia generalizadora de explicaciones a los grandes acontecimientos nacionales, profundizando en fuentes primarias de archivos históricos regionales, que han permitido desmitificar aseveraciones que antaño eran aceptadas como teorías válidas ${ }^{4}$.

2 Egracia Loyo y Anne Staples, "Fin del siglo y de un régimen”, en Historia mínima ilustrada. La educación en México, Dorothy Tanck de Estrada (México: El Colegio de México, 2011), 224-225.

3 Luz Elena Galván Lafarga, Susana Quintanilla Osorio y Clara Inés Ramírez González, Historiografía de la educación en México (México: Consejo Mexicano de Investigación Educativa, 2003), 22.

4 Como ejemplo tenemos el tema de la educación preescolar, donde hasta hace poco tiempo se creía que el nivel surge a partir de 1929, cuando se establece el primer jardín de niños oficial en la ciudad de Chihuahua. Sin embargo, gracias a las investigaciones de Martha Esther Larios Guzmán y Guillermo Hernández Orozco, hoy sabemos que el Estado tiene una rica tradición que se remonta a las últimas décadas del siglo XIX cuando surgen las primeras instituciones fundadas por las corporaciones religiosas protestantes. Ver más en Martha Esther Larios Guzmán y Guillermo Hernández Orozco. "Proceso histórico y formación docente. La Escuela Normal de Educadoras en Chihuahua, 19291934". Revista Investigación Cientifica IV , No 3 (2008), 1-14 y Martha Esther Larios Guzmán. Historia de la educación preescolar en Chibuabua, 1885-1940 (Chihuahua: Gobierno del Estado de Chihuahua / Asociación Universitaria Comunicación y Cultura, 2009), 50. Otros historiadores han estudiado el tema de la independencia nacional y encontraron que en la Villa de Chihuahua no se dio la insurgencia sino más bien la contrainsurgencia, pues en las actas de cabildo quedaron asentados los pormenores de la aprehensión de los caudillos independentistas y las acciones que tomarían para evitar brotes de insurgencia en las Provincias Internas de la Nueva Vizcaya, evidenciando diferencias contextuales de un acontecimiento de la historia nacional que en el centro del país tuvo un apoyo generalizado, mientras que en Chihuahua fue combatido abiertamente. Ver más en Rubén Beltrán Acosta. Movimiento Insurgente en Chibuabua (Chihuahua: Ayuntamiento de Chihuahua, 2010). 
En este artículo se revisan documentos de primera mano del Archivo de Concentración de Gobierno del Estado de Chihuahua (ACGECH) y de los Archivos Históricos de la Universidad Autónoma de Chihuahua (AUACH) y del Congreso del Estado (AHCECH) a fin de ofrecer un panorama amplio del desarrollo que tuvo la educación primaria a lo largo de dos períodos gubernamentales que vieron en la educación una alternativa para superar los problemas sociales del momento. Igualmente se revisa la etapa de caos en que se sumió la educación durante los primeros años de la Revolución Mexicana.

El propósito fundamental del trabajo es aportar elementos para revalorar el período porfirista como impulsor de la enseñanza primaria y del sistema de educación posprimaria -incluyendo la formación de maestros-, cuyos aportes sobrevivieron los años de conflicto e incluso fueron los elementos que permitieron la reorganización del sistema en la segunda década del siglo XX. Igualmente busca profundizar en el análisis de factores regionales que dieron un matiz diferente a los grandes procesos educativos, para romper con la tradición generalizadora de la historiografía nacional y enriquecer este campo de estudio.

Resulta una tarea esencial del investigador poder revalorar los avances que tuvo la educación en el período de bonanza económica porfiriana e identificar el estado de caos en que se sumió el sistema educativo durante el período revolucionario, sin que con ello se dejen de lado las profundas desigualdades sociales que prevalecieron durante el período estudiado y que precisamente fueron el germen que alimentó el levantamiento armado de 1910.

El procedimiento metodológico de la investigación consistió en la revisión documental de archivos y bibliografía secundaria que ayudó a definir categorías sobre las cuales versa el trabajo. A partir de ellas el investigador realizó propiamente tareas científicas de análisis e interpretación del hecho histórico, de acuerdo al marco interpretativo del autor en el que -como se sabe- no escapa la subjetividad en las afirmaciones presentadas. Ezequiel Ander-Egg señala que esta etapa corresponde precisamente a la investigación como "un procedimiento reflexivo, sistemático, controlado y crítico 
que permite descubrir nuevos hechos o datos, relaciones o leyes, en cualquier campo del conocimiento humano" 5 .

\section{LA DIFÍ́CIL TRAVESÍA POR LA EDUCACIÓN}

La educación primaria en el Estado de Chihuahua -al inicio la vida independiente- estuvo marcada por la herencia colonial en cuanto a sus métodos de enseñanza y a la inclinación religiosa, incluso en los planteles sostenidos con fondos municipales ${ }^{6}$. Las disposiciones legales orientadas a regular el servicio educativo fueron de carácter general y no integraron principios como el de gratuidad, laicismo y obligatoriedad que posteriormente fueron el eje del debate legislativo.

El contexto político, económico y social de la entidad estuvo influenciado por los acontecimientos a nivel nacional, que impusieron su propia dinámica para el funcionamiento del sistema educativo. Las pugnas entre grupos políticos locales partidarios del centralismo y del federalismo, la guerra con las tribus nómadas, el abandono que sufría el Estado con respecto al poder central y el desánimo ocasionado por la pérdida de territorio en la guerra con los Estados Unidos, obstaculizaron los proyectos educativos de largo alcance durante la segunda mitad del siglo XIX.

La enseñanza primaria tenía como deuda que los planteles contaran con maestros debidamente formados, pues desde 1843 comenzaron los intentos por establecer una Escuela Normal para Profesores -una vez que fue clausurada la Escuela Normal Lancasteriana- pero ninguno dio los resultados esperados, a pesar de que en la década de 1880 aparecieron este tipo de instituciones en la capital del país y en Estados como Veracruz y Oaxaca ${ }^{7}$.

5 Ezequiel Ander-Egg, Introducción a las técnicas de investigación social, 21a. Ed. (México: El Ateneo, 1998), 57.

6 Francisco R. Almada, Diccionario de Historia, Geografía y Biografía Chihuahuenses (Chihuahua: Universidad de Chihuahua, 1968), 178.

7 Jesús Adolfo Trujillo Holguín, Sembradores: la Normal del Estado en la historia educativa de Chihuahua (Chihuahua: Secretaría de Educación y Cultura, 2005), 29-33. 


\section{Imagen 1}

Escuela Oficial número 138 para niños en donde funcionó la Escuela Normal Lancasteriana de 1833

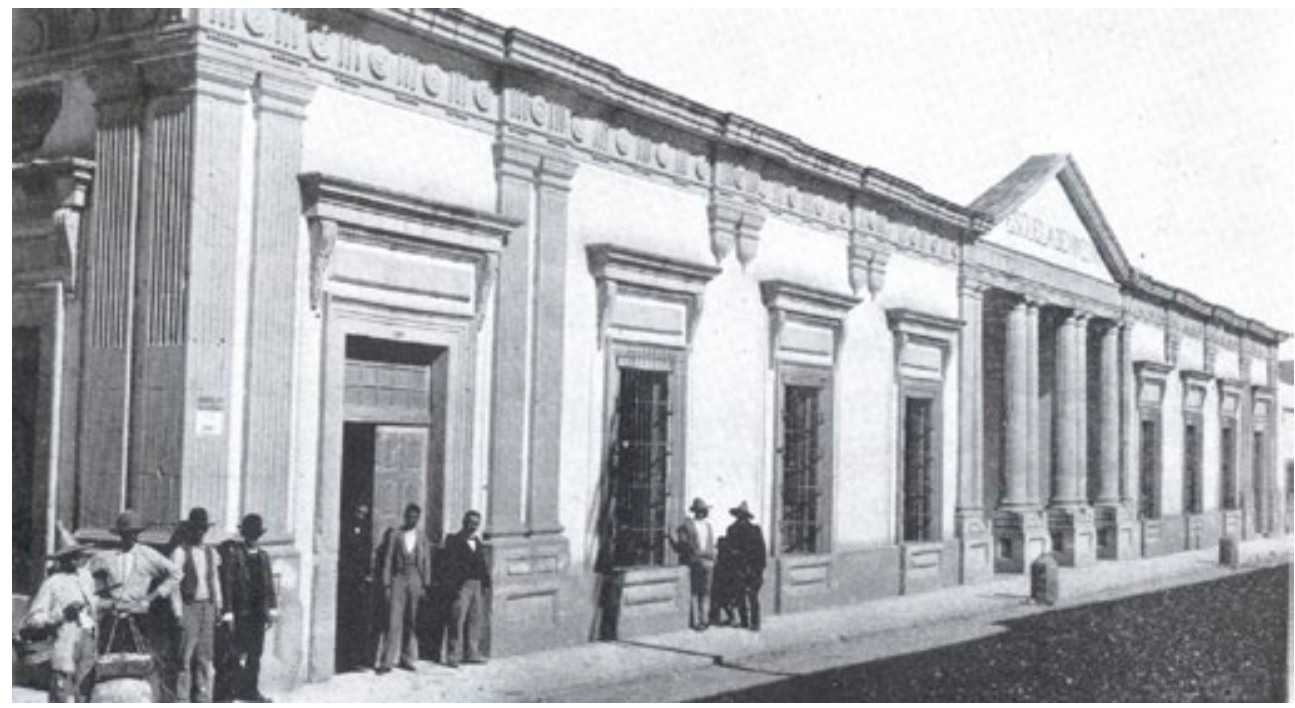

Fuente: "Álbum de enseñanza primaria del estado de Chihuahua" (Chihuahua: Imprenta El Norte S.A., 1909), en AHUACH, 37

En el aspecto normativo la educación estuvo supeditada a la reglamentación local y en la práctica se depositó la responsabilidad económica del servicio en los municipios. Esto ocasionó desigualdad de una región a otra del Estado; sin embargo se apegó a lo que estableció la Constitución Federal de 1857 en materia de libertad de enseñanza. El clero católico dejó de ejercer el control total de las instituciones educativas al separarse los asuntos de la Iglesia y el Estado con las Leyes de Reforma expedidas en los siguientes años.

La Constitución local de 1858 asentó solamente un apartado en la sección referente a las facultades y obligaciones del Congreso del Estado en donde le daba atribuciones para promover la educación, la industria pública y todos los ramos de la prosperidad del Estado ${ }^{8}$, pero sin ir más allá en lo relativo a contenidos educativos, organización de planteles o funciones de las autoridades, a pesar de que un año antes el mismo Secretario de Gobierno había comparecido ante la Legislatura local exponiendo que:

8 Enrique González Flores, Las Constituciones de Chihuahua (Chihuahua: Talleres Gráficos del Gobierno del Estado de Chihuahua, 1999), 66. 
"La penuria del Estado y la ruina más completa de los fondos municipales, de que en otra parte he informado a Vuestra Honorabilidad y de que no puede menos de resentirse en todos los ramos y objetos que debieran recibir impulso y vida de aquellos, han impedido el establecimiento y mejoras de escuelas en el grado que sería de desear y que tanto reclama el espíritu de la época...".

Es evidente que ante el clima de inestabilidad política la educación resultara afectada. La lucha armada entre liberales y conservadores, junto con las penurias que sufría el Estado, llevaron a una severa crisis económica en el erario público que obligó a las autoridades a contraer deuda. La situación se tornó grave cuando en el ámbito federal, el presidente Benito Juárez anunció la suspensión en el pago de la deuda externa, que llevó a la Segunda Intervención Francesa que se prolongó de 1862 a 1867.

La atención a la educación primaria no fue posible sin los recursos necesarios, a pesar de que en 1861 se expidió una ley reglamentaria de educación. Fueron establecidos lineamientos para la integración de una Junta Directiva de Instrucción Pública en donde vuelve a retomarse la necesidad de fundar una Escuela Normal para formar maestros ${ }^{10}$ y se incluye explícitamente la gratuidad de la educación en todo el Estado. La obligatoriedad aparece en la Ley de 8 de julio de 1875.

El progreso educativo durante el período de la República restaurada ${ }^{11}$ fue lento; sin embargo la situación comienza a tomar nuevos bríos al inicio del Porfiriato con la llegada de la prosperidad económica, que en el Estado iba a tono con la del país. Los capitales extranjeros -principalmente de Inglaterra y Estados Unidos- favorecieron el crecimiento de una pequeña oligarquía que fungía como intermediaria. Esta, además, se benefició con las políticas liberales del período reformista, relacionadas con la desamor-

9 Francisco R. Almada, Diccionario de historia..., 178.

10 Jesús Adolfo Trujillo Holguín, Sembradores..., 30.

11 El período de la República restaurada comprende desde la derrota del Segundo Imperio Mexicano -establecido por los conservadores con el apoyo de Francia y encabezado por Maximiliano de Habsburgo- en 1867 hasta el inicio del Porfiriato en 1876. 
tización de los bienes eclesiásticos. Numerosas extensiones de terreno y fincas urbanas fueron adquiridas por unas cuantas familias que entraron en el reparto de la riqueza ${ }^{12}$.

El auge económico, aunque benefició directamente a los grandes terratenientes, trajo consigo una etapa en la que fueron materializados los proyectos educativos que anteriormente habían quedado truncados por la falta de recursos. La Constitución Política del Estado de Chihuahua de 1887 recogió el ideario liberal de 1857 y estableció como garantía individual el derecho a ser instruido en los establecimientos de enseñanza sostenidos por cuenta de los fondos públicos, además de un título específico relativo a la instrucción pública en el que ya aparece la obligación del Estado de proporcionar el servicio a toda la población. En el mismo sentido fueron integrados los principios de laicidad, gratuidad, uniformidad y obligatoriedad educativa.

Es de resaltar que en un período histórico en el que interesaba construir un sentimiento nacionalista sobre el cual se edificaran las instituciones laicas, se destinó un artículo específico para normar la obligatoriedad de la lectura de las constituciones general de la República y particular del Estado en los establecimientos educativos; así como las leyes electorales relativas a cada una de ellas ${ }^{13}$. De esta manera inició un giro ideológico que cambió el culto a Dios por el culto a la Patria.

La Constitución de 1887 sentó un precedente importante para el impulso educativo. El gobernador Lauro Carrillo llevó a la práctica nuevas disposiciones e introdujo dos elementos que detonaron la expansión del sistema. El primero corresponde a la apertura de la Cátedra de Pedagogía que explica el rápido crecimiento del número de escuelas de enseñanza primaria

12 Luis Aboites señala que durante el Porfiriato hubo grandes latifundios de empresarios extranjeros y locales, destacando el de la México Northwestern Railway de 1 millón de hectáreas, el de la Bavícora del periodista Randolph Hearst de 350 mil hectáreas y el de T. O. Riverside de 500 mil hectáreas. En la lista de terratenientes locales enumera a Luis Terrazas con 2 millones de hectáreas, los Zuloaga con 600 mil hectáreas en Bustillos y Satevó, los Luján con 200 mil hectáreas en Santa Gertrudis, entre otros. Ver Luis Aboites, Breve historia de Chihuahua (México: Fondo de Cultura Económica/El Colegio de México, 1994), 108-119.

13 Enrique González Flores, Las Constituciones..., 106. 
que pasó de 102 en 1879 a 270 en 1891; es decir, un aumento de 14 planteles por año. El segundo fue la invitación que hizo al pedagogo alemán Enrique Laubscher para que iniciara la reforma educativa en el Estado e introdujera métodos modernos como la enseñanza simultánea de lectura y escritura $^{14}$ que estaba popularizando en Veracruz ${ }^{15}$. Francisco Alberto Pérez Piñón señala que como resultado del diagnóstico de Laubscher “[...] se planteó la necesidad de mejorar las escuelas mientras, cualitativamente, se valoraba la necesidad de formar profesores en los métodos modernos que se tradujeran en logros efectivos de los alumnos en las escuelas públicas"16.

Podemos inferir que durante los primeros años del Porfiriato ocurrió un cambio importante en el imaginario educativo. Aparecen nuevos elementos en el debate que hablan de una preocupación que no se limitaba al aspecto cuantitativo del sistema (apertura de más escuelas, creación de nuevas estructuras operativas, fundación de una Escuela Normal, entre otros elementos) sino de calidad del servicio, reflejada en la introducción de nuevos métodos de enseñanza en contraposición a las prácticas heredadas del lancasterianismo, tales como uso de castigos físicos y aprendizaje basado en la memorización.

En suma, desde la llegada del período independiente transcurrieron seis décadas en las que hubo avances y retrocesos que contribuyeron a la formación de un ideario educativo en la clase gobernante y aunque no logró materializarse en acciones trascendentes y duraderas, sí generó un importante movimiento a favor de la educación pública, que solamente debía

14 La enseñanza de la lectura y la escritura a lo largo del siglo XIX se realizó con métodos en los que predominaba el deletreo, luego aprendían sílabas y posteriormente podían leer palabras y frases completas; sin embargo la enseñanza era memorística y ocurría el caso en donde el alumno aprendía a leer pero no a escribir, es decir, los procesos se llevaban de manera separada. Para ver más sobre el tema puede consultarse Francisco Alberto Pérez Piñón, Guillermo Hernández Orozco, y Jesús Adolfo Trujillo Holguín, "Aprender a leer y escribir en Chihuahua: siglo XIX," IE Revista de Investigación Educativa de la REDIECH (Red de Investigadores Educativos Chihuahua), $n^{\circ} 9$ (Octubre 2014): $35-42$.

15 Egracia Loyo y Anne Staples, "Fin del siglo...", 198.

16 Francisco Alberto Pérez Piñón, Contribución educacional de la Escuela Normal del Estado de Chibuabua en el periodo de la Revolución Mexicana (Chihuahua: Sindicato del Personal Académico de la UACH/Universidad Autónoma de Chihuahua, 2007), 27. 
esperar las condiciones adecuadas para que prosperara. Precisamente una coyuntura política permitió la llegada al poder de un personaje identificado con la causa educativa, que permaneció en el cargo durante el tiempo suficiente para materializar los proyectos.

\section{El ARRIBO AL PODER DEL GOBERNANTE EDUCADOR}

La entrada del coronel Miguel Ahumada ${ }^{17}$ a la gubernatura estatal representó el inicio de uno de los períodos de mayor auge para la educación primaria, no solamente por las disposiciones legales que garantizaron la organización del sistema, sino por acciones que materializaron los proyectos educativos. Quizás el más significativo fue la invitación que hizo a un grupo de profesores egresados de la Normal de Jalapa, discípulos del pedagogo Enrique C. Rébsamen, que culminaron la obra educativa iniciada por Laubscher y que detonó el establecimiento definitivo de la Escuela Normal para Profesores en 1906.

El juicio que la historia ha realizado sobre las aportaciones de Miguel Ahumada se contrapone entre quienes le dan el calificativo de "gobernante educador"18 por la gran cantidad de innovaciones que hubo en todo el sistema, la apertura de nuevas instituciones, la construcción y reparación de edificios escolares y las becas que otorgó para que los jóvenes acudieran a realizar estudios en otras partes del país y del extranjero; y los que lo denominan el "asesino de la educación superior"19 porque suprimió dichos estudios en el Instituto Científico y Literario por lo onerosos que resultaban para el Estado, dejando únicamente la Escuela Preparatoria. Lo cierto es que la educación experimentó una notable mejora. Concedió especial

17 Miguel Ahumada fue un militar y político colimense. Nació el 29 de septiembre de 1844 y en su juventud desempeñó el oficio de carpintero y guardia aduanal. Combatió en las fuerzas patrióticas contra los invasores franceses y a partir de 1870 comenzó a ocupar cargos políticos hasta que en 1876 resultó electo diputado local en Colima. Llegó a Chihuahua a ocupar el cargo de jefe de la III zona de la Gendarmería Fiscal en 1886 y en 1892 fue electo Gobernador del Estado. Ver más en Ulises Irigoyen, Miguel Ahumada, gobernante educador (Chihuahua: Gobierno del Estado de Chihuahua, 2001), 1-2.

15418 Ulises Irigoyen, Miguel Ahumada..., 3-6.

19 Guillermo Hernández Orozco, El Instituto Científico y Literario de Chihuahua 1850-1900 (Chihuahua: Universidad Autónoma de Chihuahua/Sindicato del Personal Académico de la UACH, 1999), 171. 
interés a los temas prioritarios en la agenda educativa de aquella época y el rasgo distintivo fue la apertura y cierre de su gobierno con obras educativas. El profesor normalista Alberto Vicarte, en un telegrama enviado el 27 de febrero de 1903, dio cuenta al mandatario de los pormenores de la representación que ejerció en su nombre para la inauguración del edificio de la Escuela Superior de Niñas de Hidalgo del Parral, señalando que "El primer acto trascendental de su gobierno a fines de 1892, fue la inauguración del edificio escolar destinado a la Escuela Anexa a la Normal, y el último, la inauguración de esta escuela de Hidalgo del Parral"20.

La vocación educadora de Miguel Ahumada se expresa de manera fiel en las memorias de sus informes de gobierno. Precisamente al finalizar su primer mandato, en 1896, expresa ante el Congreso del Estado que:

"Porque tengo la convicción de que para el progreso y bienestar de los pueblos, se necesita antes que todo formar ciudadanos, es decir, enseñar a los hombres sus deberes políticos y sociales; las ventajas del saber, cualesquiera que sean las ocupaciones a que lleguen a dedicarse; los males de la ociosidad, los peligros de los vicios; y en fin, porque la experiencia me ha demostrado, que las escuelas oficiales además de cultura dan a los pueblos unidad y fuerza, pues poniendo a los hombres de todas las clases sociales en contacto, forzosamente hacen nacer entre ellos esas relaciones de la infancia tan duraderas, que producen la emulación y destruyen los odios creados por la posición y la riqueza. Por todo esto, repito, es por lo que, sin desatender los demás ramos de la administración, pues todos ofrecen igual interés público, he consagrado a la instrucción un cuidado especial; y sin embargo, aún no estoy satisfecho, porque aunque rápidos y sensibles los adelantos en el estado, no están a la altura en que mi empeño quisiera verlos colocados"21.

20 Ulises Irigoyen, Miguel Ahumada..., 3-6.

21 "Informe del Gobernador Constitucional coronel Miguel Ahumada al H. Congreso del Estado" (Chihuahua, 1896), en Archivo Histórico de la Universidad Autónoma de Chihuahua (AHUACH), Sección Educación, Fondo Porfiriato, Documento Álbum de enseñanza primaria. 


\section{Imagen 2}

Coronel Miguel Ahumada, gobernador del estado de Chihuahua de 1892 a 1903

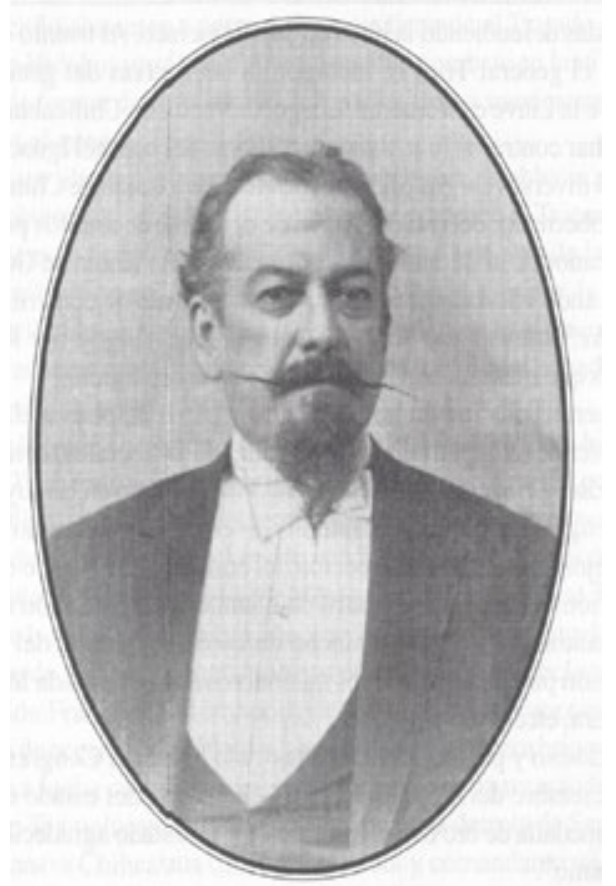

Fuente: Joaquín Márquez Montiel, Hombres célebres de Chihuahua. 2a. Ed.

(Chihuahua: Gobierno del Estado de Chihuahua, 2004), 282

En las palabras del Gobernador puede apreciarse un discurso educativo de carácter popular que coincide con la naturaleza de las acciones desarrolladas durante su administración. La aspiración de una instrucción encaminada a romper barreras de clase no necesariamente fue producto exclusivo de la Revolución Mexicana, sino que se fraguó como ideario incluso durante el Porfiriato, pero con un avance muy lento. Lo mismo ocurrió con la visión acerca del ámbito de competencia del Estado como componente de equilibrio entre las clases sociales, que no figuró exclusivamente en el discurso, pues la decisión de suprimir la educación superior, obedeció a una tendencia emancipadora. En el mismo informe de 1896 justificó su política educativa argumentando que:

"Una de las tantas economías introducidas para atender a la instrucción primaria, fue la supresión de la enseñanza superior o profesional, iniciada por el Ejecutivo [...] entre otras considera- 
ciones, por la de que la obligación de dar profesión pertenece a la familia más que a los gobiernos, que cumplen su misión proporcionando a la juventud, los conocimientos elementales que preparen su inteligencia y actividad, para dedicarse a los trabajos a que por sus condiciones sea llamada"22.

El texto anterior orientaba la filosofía educativa hacia el establecimiento de las bases para el desarrollo de la sociedad, que se encontraba en la instrucción primaria, aunque durante esta administración fueron inauguradas las Escuelas Industrial para Señoritas y de Artes y Oficios que corresponden al nivel posprimario, desarrollado ampliamente durante la administración del gobernador Enrique C. Creel.

El primer paso en la transformación educativa consistió en establecer los fundamentos normativos mediante la Ley Reglamentaria de la Instrucción Pública el 17 de diciembre de 1892, que ratificó el principio de obligatoriedad de la enseñanza elemental vigente desde 1875. Posteriormente aparece la Ley de 15 de julio de 1893 en donde declara gratuita, laica y obligatoria la enseñanza; crea la Junta Superior de Instrucción Pública y las Juntas Inferiores en cada municipio. Además hay un adelanto muy importante porque fijó por primera vez el uso de textos escolares e incluyó la educación para párvulos ${ }^{23}$. Esta última se detonó en la ciudad de Chihuahua con la llegada de los grupos protestantes que arribaron en $1882^{24}$.

En los contenidos educativos correspondió a este período la implantación del régimen militar en las escuelas para varones, con la intención de fo-

22 "Informe del Gobernador Constitucional coronel Miguel Ahumada al H. Congreso del Estado" (Chihuahua, 1896), en AHUACH, Sección Educación, Fondo Porfiriato, Documeto Álbum de enseñanza primaria.

23 La educación para párvulos se refiere a la que estaba destinada a los niños menores de 7 años. Véase Luz Elena Galván Lafarga y Alejandra Zúñiga, "De las escuelas de párvulos al preescolar. Una historia por contar", Diccionario de historia de la educación en México. 2002. http://biblioweb.tic.unam. $\mathrm{mx} /$ diccionario/htm/articulos/sec_25.htm (último acceso: 7 de marzo de 2016).

24 Para ver más sobre los grupos protestantes en Chihuahua puede consultarse a Rubén Beltrán Acosta, "La llegada del protestantismo a la ciudad de Chihuahua", Vol. I, de Chihuahua, Horizontes de su historia y su cultura, de Jesús Vargas Valdés (coord.). México: Grupo Editorial Milenio, 2010, 132 147. 
mentar el patriotismo y la obediencia a las autoridades ${ }^{25}$; destacando la de ciudad Guerrero, dirigida por el maestro Mariano Irigoyen, que hacía gala de la preparación militar de los alumnos, durante los desfiles organizados con motivo de las fiestas patrias ${ }^{26}$.

\section{Imagen 3}

Alumnos de la Escuela Anexa a la Normal del Estado en ejercicios militares

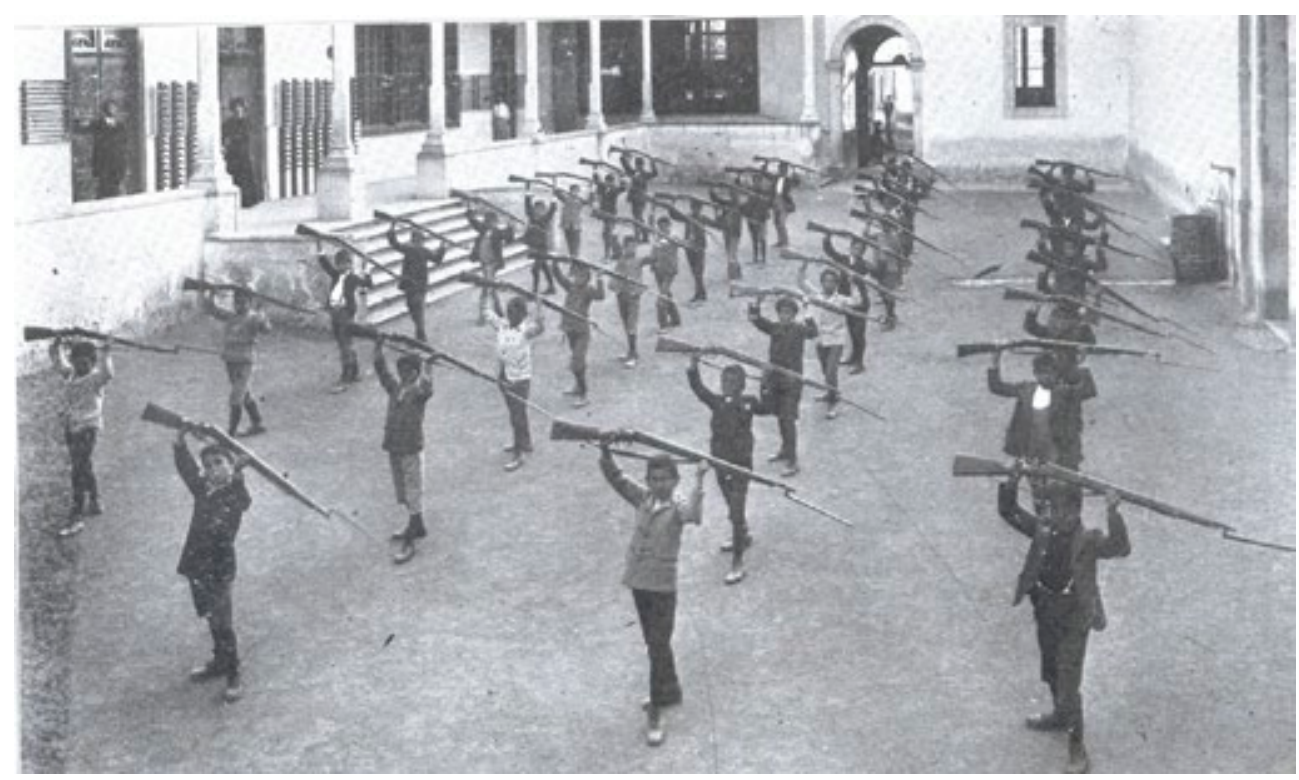

Fuente: "Álbum de enseñanza primaria del estado de Chihuahua" (Chihuahua: Imprenta El Norte S.A., 1909), en AHUACH, 22

Los cambios y las innovaciones implementadas en el sistema educativo nos hablan de la presencia de otras corrientes de pensamiento pedagógico. La cercanía con Estados Unidos facilitó cierta influencia en el sistema educativo, en tanto que el interés por allegarse de personas que estaban formadas en las técnicas didácticas más avanzadas favoreció el intercambio con la capital del país y con el Estado de Veracruz. Esta última entidad recibió el influjo pedagógico europeo y precisamente en el gobierno de Ahumada llegaron los discípulos del afamado Enrique C. Rébsamen, quien fundó la Escuela Normal de Profesores de Jalapa en 1887. Loyo y

25 Francisco R. Almada, Diccionario de Historia..., 181.

26 Jesús Vargas Valdés, Mariano Irigoyen Escontrías, una vida por la educación (Chihuahua: Gobierno del Estado de Chihuahua, 2002), 13. 
Staples señalan que esta institución "Fue semillero de maestros y ocasionó una verdadera dispersión de educadores" 27 , lo cual es perfectamente aplicable en Chihuahua ya que a partir de 1894 comenzaron a llegar sus alumnos más aventajados: Alberto Vicarte, Abel S. Rodríguez, Gonzalo Reyes, Joaquín Barcárcel, Manuel E. Rosas, Miguel A. López, Ricardo C. Campillo, Ernesto Alconedo, Leopoldo Rodríguez Calderón, Víctor N. Lara, Rodolfo González Llorca, entre otros ${ }^{28}$. Al paso de los años estos personajes fueron soporte del sistema educativo al desempeñarse como autoridades o directivos de planteles como el Instituto Científico y Literario.

La presencia de maestros formados en un ambiente de intensa actividad educativa favoreció que tanto en la normatividad como en los proyectos de gobierno prevaleciera la orientación pedagógica que permeaba a nivel nacional. Algunas resoluciones del Primero y Segundo Congreso de Instrucción Pública se identifican perfectamente en las disposiciones locales: la educación laica, el uso de textos escolares, la uniformidad de la enseñanza, eliminación del método lancasteriano, entre otras. Sin embargo hubo recomendaciones que el gobierno estatal no acató y más bien las adecuó a las circunstancias locales como fue el caso de los ejercicios militares que en el Segundo Congreso - celebrado del 1 de diciembre de 1890 al 28 de febrero de 1891- ${ }^{29}$ fueron desterrados y en Chihuahua continuaron en práctica.

Durante el segundo período gubernamental de Miguel Ahumada se expidió la Ley de Instrucción Pública de 1897, en la que ratificaron algunos principios de la anterior, y se incluyeron aspectos que reflejan la influencia profesional ejercida por los maestros foráneos que estaban enriqueciendo el ambiente pedagógico de Chihuahua. Esta vez se decretó la preferencia en las contrataciones para los maestros titulados, se declaró altamente honroso servir al magisterio y apareció por primera vez el uso de recompensas para los docentes como reconocimiento a sus años de servicio. Es-

27 Egracia Loyo y Anne Staples, “Fin del siglo...", 198.

28 Irigoyen, Ulises. Miguel Ahumada..., 9-10.

29 Egracia Loyo y Anne Staples, "Fin del siglo...", 207. 
tos fueron discutidos ampliamente en el Primer Congreso de Instrucción Pública, celebrado de 1889-1890.

La transición educativa hacia la modernización fue notable durante el gobierno de Miguel Ahumada, aunque hubo aspectos estructurales que minaron los avances a la llegada del movimiento revolucionario. Uno de ellos fue la excesiva centralización que se ejerció en un sistema que funcionaba con cierto grado de autonomía por parte de los municipios. Estos últimos eran quienes tenían la carga económica, mientras que el Estado establecía una estructura administrativa que ejercía el control en todos los aspectos operativos, lo que a la larga se interpretó como una intromisión. De cualquier forma los adelantos continuaron y la llegada de una nueva administración, que sigue en la tónica de fomentar la educación en todos sus niveles, permitió un desarrollo que tendría su punto culmen hasta 1909, a la víspera del movimiento revolucionario.

\section{La educación durante el gobierno de Enrique C. Creel}

La expansión de la educación en el Estado continuó en la etapa que va de 1904 a 1911, cuando estuvo como gobernador interino y constitucional del Estado el economista Enrique C. Creel ${ }^{30}$. Bajo su mandato impulsó fuertemente la educación pública y finalmente es quien coronó el viejo anhelo de contar con una Escuela Normal para Profesores en la ciudad de Chihuahua, que inauguró el 2 de enero de 1906. Además consolidó la educación posprimaria al establecer la Escuela Comercial y la Escuela Particular de Agricultura de los Hermanos Escobar.

30 Enrique C. Creel ocupó la gubernatura del Estado de Chihuahua de manera interina de 1904 a 1906 y en el período constitucional de 1907 a 1911. Fue un notable economista que se dedicó a los negocios desde muy temprana edad, cuando tuvo que ocuparse de los asuntos de su padre. Ocupó puestos en instituciones bancarias y económicas como el Banco Minero de Paso del Norte, Banco Minero de Chihuahua, Comisión de Cambios, Consejo de Ferrocarriles del Estado de Yucatán, Asociación de Banqueros de la República Mexicana, Compañía Kansas City, entre otras. En la política inició como regidor y síndico del ayuntamiento de Chihuahua hasta convertirse en embajador en Washington y Secretario de Relaciones Exteriores en el gabinete porfirista. El historiador Jesús Vargas Valdés sostiene la teoría de que era una de las alternativas del general Porfirio Díaz para sucederlo en el poder, a juzgar por las tareas que le fue encomendando a partir de 1904. 

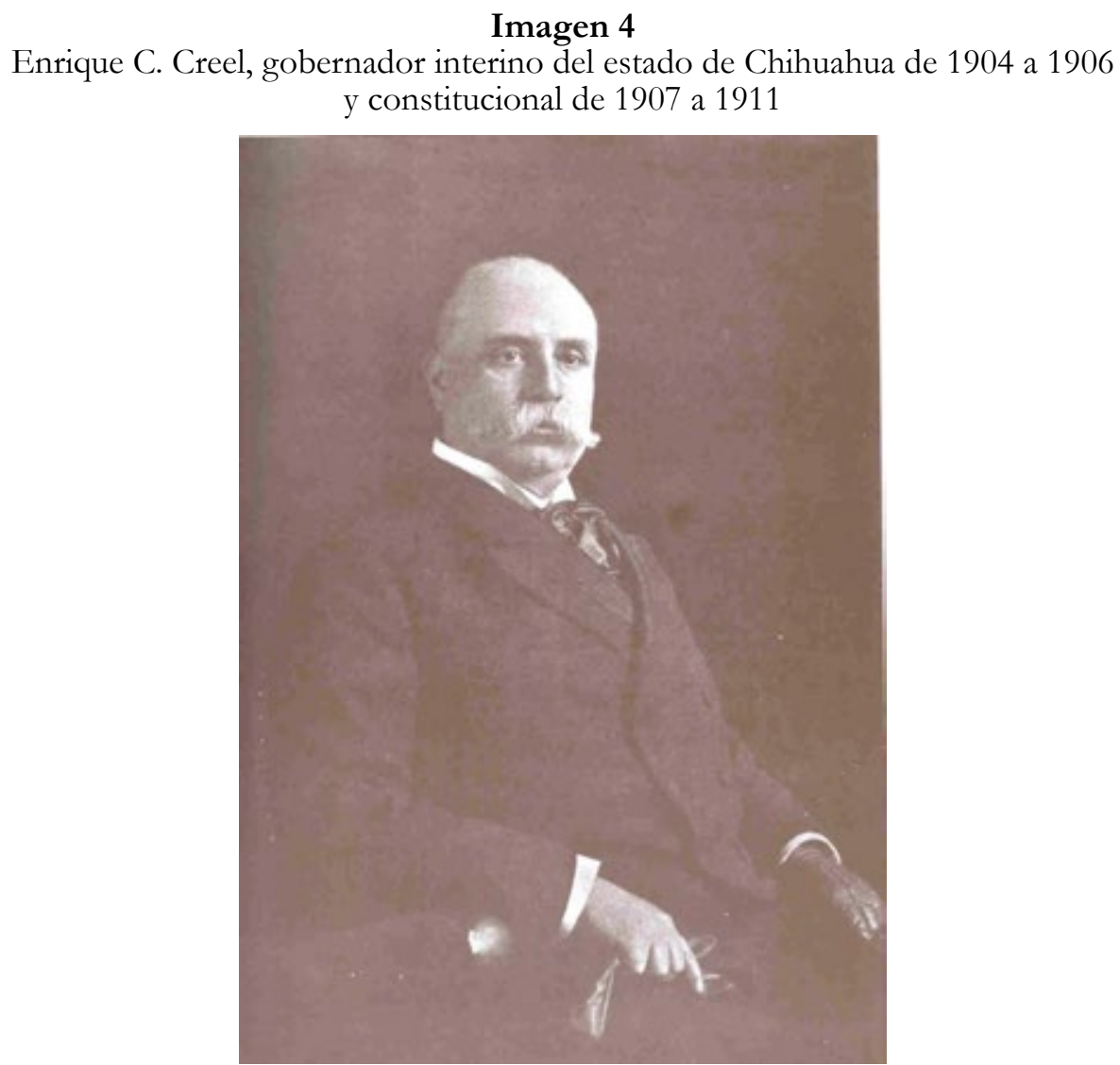

Fuente: “Álbum de enseñanza primaria...”, 2

En los informes de gobierno y memorias de la época quedó perfectamente documentado el estado en que se encontraba la educación en Chihuahua. En estudios elaborados en el ámbito nacional puede constatarse ese período fructífero catalogado como el más importante del país por el presupuesto educativo, la asistencia media de alumnos al año y el número de planteles en operación ${ }^{31}$.

En el primer período de gobierno de Enrique Creel destaca la prevalencia en el discurso político de la prioridad en que colocaba a la educación. En el informe que leyó al Congreso del Estado, el 16 de septiembre de 1904, refiere que "De los ramos de la administración pública ninguno ha preocu-

31 Egracia Loyo y Anne Staples, "Fin del siglo...”, 224-225. 
pado tanto la atención $[\ldots]$ como el de la enseñanza popular porque $[\ldots]$ la ilustración de las masas es la que dá mayor prestigio á un pueblo"32. Este tipo de afirmaciones fueron una constante hasta el final de su gobierno.

La magnitud del crecimiento educativo en ese período fue importante. Entre 1903 y 1904 se crearon dos Inspecciones de Zona y 45 escuelas $^{33}$, en las que hubo inversiones importantes para dotarlas de mobiliario, útiles escolares e infraestructura que respondiera a las medidas higiénicas y de seguridad anteriormente discutidas en los Congresos de Instrucción Pública. Para los siguientes años el crecimiento de planteles oficiales se sostuvo, como aparece en la Tabla 1.

Tabla 1. Escuelas de instrucción primaria existentes en el cuatrienio 1904-1908

\begin{tabular}{|l|r|r|r|r|c|}
\hline Tipo de escuelas / año & 1904-1905 & 1905-1906 & 1907 & 1908 & Aumento \\
\hline Primera clase & 13 & 20 & 21 & 21 & 8 \\
\hline Segunda clase & 41 & 41 & 45 & 45 & 4 \\
\hline Tercera clase & 148 & 150 & 148 & 155 & 7 \\
\hline Adultos & 6 & 3 & 3 & 3 & -3 \\
\hline Párvulos & 1 & 1 & 1 & 1 & 0 \\
\hline Subvencionadas & 12 & 87 & 120 & 133 & 121 \\
\hline Incorporadas & 3 & 7 & 3 & 3 & 0 \\
\hline Privadas & 19 & 8 & 36 & 38 & 19 \\
\hline Totales & 243 & 317 & 377 & 399 & 156 \\
\hline
\end{tabular}

Fuente: "Álbum de enseñanza primaria...", 11-38

La apertura de planteles refleja una atención creciente a la demanda educativa que se extendió también a las comunidades con escasa matrícula, a través de las escuelas subvencionadas. Estas operaban con un subsidio mensual del gobierno y con la dotación de libros y útiles escolares. Funcionaban en los lugares donde los recursos no alcanzaban para sostener una escuela oficial.

32 “«Informe leído el 16 de septiembre de 1904 por el gobernador interino constitucional del Estado de Chihuahua C. Enrique C. Creel, en la apertura del primer período de sesiones ordinarias del XXIV Congreso»" (Chihuahua, 16 de septiembre de 1904), en Archivo Histórico del Congreso del Estado de Chihuahua (AHCECH), Sección Informes de Gobierno, Fondo Porfiriato.

33 "Álbum de enseñanza primaria del Estado de Chihuahua" (Chihuahua, 1909) en AHUACH, Sección Educación, Fondo Enseñanza primaria, Documento Álbum de enseñanza primaria. 
Es importante considerar que en la administración del gobernador Creel se buscó atacar los problemas educativos de fondo, considerando que una de las limitantes más fuertes era la ausencia de maestros capacitados; la obra más trascendente fue la expedición de la Ley de Enseñanza Normal de 24 de abril de 1905, que dio la posibilidad para fundar la Escuela Normal. De esta manera ya no hubo impedimentos para que la Institución abriera sus puertas el 2 de enero de 1906, con una matrícula de 12 estudiantes ${ }^{34}$.

Al cierre de la administración del gobernador Enrique C. Creel resaltaron dos rubros que fueron la constante en el período Porfirista. El primero corresponde a la inversión en edificios escolares y el segundo a la preparación de profesores de acuerdo a los avances pedagógicos del momento. En el informe de gobierno, presentado ante el Congreso del Estado el 1 de junio de 1909, destacó la construcción y reparación de escuelas en poblaciones a lo largo y ancho del Estado, dotación de materiales educativos e inversión en planteles del nivel posprimario ${ }^{35}$; sin embargo el resultado de la nueva era en la formación de maestros se reflejó más tarde, durante el movimiento revolucionario.

\section{La llegada del caos Revolucionario}

El esplendor alcanzado hasta la parte final de la administración del gobernador Creel fue truncado de golpe con la llegada del movimiento armado de 1910. Algunas escuelas cerraron a causa de la inseguridad, falta de pagos a los docentes e inestabilidad en el nombramiento de autoridades educativas. Trujillo Holguín ejemplifica este último fenómeno con el Instituto Científico y Literario "que contó con la dirección del doctor Miguel Márquez durante 18 años ininterrumpidos durante el Porfiriato, de 1893 a

34 Francisco R. Almada, Guía histórica de la ciudad de Chihuahua (Chihuahua: Gobierno del Estado de Chihuahua, 1981), 237-238.

35 "Informe leído por el gobernador constitucional del Estado C. Enrique C. Creel el día $1^{\circ}$ de junio de 1909 en la apertura del $2^{\circ}$ período ordinario de sesiones de la XXVI correspondiente al último año de su ejercicio" (Chihuahua, 1909), en AHCECH, Sección Informes de Gobierno, Fondo Porfiriato, Documento 17. 
1911; mientras que en un período similar, de 1911 a 1929, pasaron por la dirección del plantel una docena de maestros"36.

Tan solo en los primeros años de revuelta fue evidente el grado de descomposición del sistema educativo. El panorama para 1911 era muy distinto a los años de esplendor Porfirista y el mismo gobernador Abraham González señala en su informe del 30 de julio de 1912, al Congreso del Estado, la situación en que se encontraba la educación. En el caso del Instituto Científico y Literario resalta los cambios frecuentes de directivos y maestros, los que influyen desfavorablemente en el aprovechamiento de los alumnos, en tanto que en la instrucción primaria evidencia desorganización. Al respecto asienta que "La Instrucción Primaria ha sufrido como la anterior, graves trastornos durante estos últimos meses de agitación y de desorden. Excepto en esta capital, las escuelas han tropezado con muchas dificultades en todo el resto del Estado" 37 .

La estabilidad económica se vio trastocada, de manera que fue constante la suspensión de pagos a los maestros. El gobernador González dio cuenta en el mismo informe de 1912, que tanto en la capital como fuera de ella se adeudaban sueldos y había numerosas vacantes a consecuencia del movimiento revolucionario. Incluso existió el problema de la duplicidad de funciones debido a que los grupos guerrilleros que tomaban el control en alguna región del Estado, realizaban sus propios nombramientos y al recuperar el control, el bando contrario desconocía muchas de las acciones emprendidas.

En los expedientes de maestros son comunes las cartas de renuncia, que no se presentaron con tal magnitud durante el Porfiriato. Por ejemplo, la maestra Hipólita Martínez, directora de la Escuela Oficial número 56 menciona en una carta del 30 de agosto de 1912 que "Con motivo de la

36 Jesús Adolfo Trujillo Holguín, La educación socialista en Chihuahua 1934-1940, una mirada desde la Escuela Normal del Estado (Chihuahua: Universidad Autónoma de Chihuahua/Sindicato del Personal Académico de la UACH, 2015), 66-67.

37 “«Informe del Gobernador Constitucional C. Abraham González al H. Congreso del Estado»". (Chihuahua: Imprenta del Gobierno a cargo de Gilberto A. De la Garza, 1912) en AHCECH, Sección Informes de Gobienro, Fondo Porfiriato, Documento 11. 
inseguridad que reina en Julimes por las visitas frecuentes de los revolucionarios a esos lugares, me veo precisada a renunciar a mi empleo" 38 . La situación fue más dramática en las poblaciones rurales, mientras que en las escuelas urbanas hubo mayor estabilidad.

Uno de los aspectos que facilitó el desarrollo económico en las décadas anteriores fue el largo período de paz. Durante el movimiento revolucionario el panorama cambió drásticamente, lo que dificultó la marcha normal de la educación. El pago de la nómina ocurría con muchas dificultades; algunas veces por la escasa recaudación y otras por las revueltas, principalmente en los municipios con presencia de los grupos villistas y orozquistas ${ }^{39}$. En el mismo año de 1912, por ejemplo, el director de Instrucción Primaria informa al profesor Germán N. Durán que:

"sus recibos fueron cobrados hoy mismo en la Tesorería General del Estado, pero no hay giros telegráficos para ese punto, ni por San Isidro, pensé mandárselos por Exprés pero no hay directo para ese lugar y para San Isidro sí, mas no quieren recibir dinero, porque dicen han suspendido las remesas por el rumbo del Noroeste" ${ }^{\prime 4}$.

De esta manera el movimiento revolucionario arrasó con los grandes logros de las administraciones Porfirianas y tuvieron que pasar muchos años para que -en primer lugar- fuera definido un proyecto ideológico que dejara atrás las concepciones del régimen y posteriormente hubiera una estructura administrativa, recursos económicos e infraestructura adecuada para consolidarlo. En este renglón fueron retomadas las aportaciones existentes y la Escuela Normal se convirtió en uno de los pilares más importantes. Jesús Vargas Valdés señala al respecto que:

38 "Expediente de la profesora Hipólita Martínez", (Chihuahua, 30 de agosto de 1912), en Archivo de Concentración de Gobierno del Estado de Chihuahua (ACGECH), Sección Expedientes de maestros, Fondo Maestros rurales, Caja 5, Expediente 105, Documento 2.

39 Los grupos revolucionarios villistas corresponden a los que comandaba el general Francisco Villa y los orozquistas a los que lideraba Pascual Orozco en el municipio de Guerrero.

40 "Expediente del profesor Germán N. Durán" (Chihuahua, 31 de agosto de 1912) en ACGECH, Sección Expedientes de maestros, Fondo Maestros rurales, Caja 6a , Expediente 26, Documento 3. 
"En la ciudad de Chihuahua no procede la aseveración de que la educación para el pueblo llegó con la revolución, o de que antes de 1910 solo unos cuantos privilegiados tenían acceso a la escuela. Afirmamos que desde la década de 1890 se emprendió un programa educativo encaminado a llevar la educación al mayor número de niños, y no fueron los privilegios sociales o factores ideológicos los que impidieron un mayor avance, sino las condiciones de inestabilidad política y económica, y, principalmente, la falta de profesores"

Como balance general tenemos que la Revolución Mexicana se erigió como movimiento legítimo a favor de la justicia social, sin embargo en el aspecto educativo no podemos desconocer que generó un grave desorden, que efectivamente permitió la llegada de un sistema con orientación popular, pero no necesariamente fraguado en el período posrevolucionario. El Porfiriato marcó una ruta a seguir y ello lo constatan el número de escuelas oficiales que aún funcionan en el Estado de Chihuahua y que datan de aquella época ${ }^{42}$.

\section{Conclusiones}

El período analizado nos permite deducir que las últimas dos décadas del régimen Porfirista -de 1892 a 1911- significaron una etapa de esplendor educativo para el Estado de Chihuahua, materializada en las disposiciones normativas que suprimieron los métodos tradicionales de enseñanza y establecieron las bases para el surgimiento del moderno sistema educativo, con planteles que respondieron a las necesidades del desarrollo económico y social del momento.

41 Jesús Vargas Valdés, "La utopía de la educación porfiriana en Chihuahua y los afanes para fundar una escuela de maestros. 1890-1910", en Chihuahua, horizontes de su historia y su cultura, Jesús Vargas Valdés (coord.) (México: Grupo Editorial Milenio, 2010), 104-121.

16642 Los principales núcleos urbanos del Estado de Chihuahua cuentan con por lo menos una Escuela Primaria Oficial que data de la época Porfiriana y en ellas hay documentación que evidencia que no necesariamente era un servicio reservado para los niños de clase económica alta. Ver más en Guillermo Hernández Orozco, El Instituto... 
El establecimiento de instituciones como la Escuela Industrial para Señoritas, Escuela de Artes y Oficios, Escuela Comercial, Escuela Normal del Estado y Escuela Particular de Agricultura de los Hermanos Escobar fueron un pilar para el sistema educativo estatal que permaneció de pie en las décadas posteriores al movimiento revolucionario de 1910. Su influencia fue tal que, por ejemplo, la Normal del Estado funciona ininterrumpidamente hasta la fecha.

El período de transición de la educación en la época porfiriana al proyecto revolucionario significó solamente el desmoronamiento de un proyecto ideológico y la imposición de uno nuevo, basado en la justicia social. Sin embargo su edificación fue posible gracias a los logros alcanzados en el período que se criticaba.

La indagación en los antecedentes del sistema educativo estatal permite revalorar la vocación educativa del Estado de Chihuahua, la capacidad innovadora frente al contexto nacional y las expectativas de mejora, a partir del reconocimiento de los aciertos y desaciertos que marcaron el rumbo para el actual sistema.

\section{Bibliografía}

\section{Fuentes primarias}

Archivo de Concentración de Gobierno del Estado de Chihuahua (ACGECH). Chihuahua-México. Sección Expedientes de profesores, Fondo maestros rurales.

Archivo Histórico del Congreso del Estado de Chihuahua (AHCECH). Chihuahua-México. Sección Informes de Gobierno, Fondo Porfiriato.

Archivo Histórico de la Universidad Autónoma de Chihuahua (AHUACH). Chihuahua-México. Sección Educación, Fondo enseñanza primaria.

\section{Fuentes secundarias}

Almada, Francisco R. Diccionario de Historia, Geografía y Biografía Chihuahuenses. 2a. Ed. Chihuahua: Universidad de Chihuahua, 1968.

- Guía histórica de la ciudad de Chihuahua. Chihuahua: Gobierno del Estado de Chihuahua, 1981. 
Aboites, Luis. Breve historia de Chihuahua. México: Fondo de Cultura Económica/El Colegio de México, 1994.

Beltrán Acosta, Rubén. La llegada del protestantismo a la ciudad de Chihuahua. Vol. I, de Chihuahua, horizontes de su historia y su cultura, (coord.) Jesús Vargas Valdés. México: Grupo Editorial Milenio, 2010. Beltrán Acosta, Rubén. Movimiento Insurgente en Chihuahua. Chihuahua: Ayuntamiento de Chihuahua, 2010.

Galván Lafarga, Luz Elena, y Alejandra Zúñiga. “«De las escuelas de párvulos al preescolar. Una historia por contar»”. Diccionario de historia de la educación en México. 2002. http:/ / biblioweb.tic.unam.mx/ diccionario/htm/articulos/sec_25.htm (último acceso: 7 de marzo de 2016).

Galván Lafarga, Luz Elena, Susana Quintanilla Osorio y Clara Inés Ramírez González. Historiografía de la educación en México. México: Consejo Mexicano de Investigación Educativa, 2003.

González Flores, Enrique. Las Constituciones de Chihuahua. Chihuahua: Talleres Gráficos del Gobierno del Estado de Chihuahua, 1999.

Hernández Orozco, Guillermo. El Instituto Científico y Literario de Chihuahua 1850-1900. Chihuahua: Universidad Autónoma de Chihuahua/Sindicato del Personal Académico de la UACH, 1999.

Irigoyen, Ulises. Miguel Ahumada, gobernante educador. Chihuahua: Gobierno del Estado de Chihuahua, 2001.

Larios Guzmán, Martha Esther. Historia de la educación preescolar en Chihuahua, 1885-1940. Chihuahua: Gobierno del Estado de Chihuahua/Asociación Universitaria Comunicación y Cultura, 2009.

Larios Guzmán, Martha Esther, y Guillermo Hernández Orozco. “«Proceso histórico y formación docente. La Escuela Normal de Educadoras en Chihuahua, 1929-1934»»". Revista Investigación Científica IV, $\mathrm{N}^{\mathrm{o}} 3$ (2008): 1-14.

Loyo, Egracia, y Anne Staples. “«Fin de siglo y de un régimen»”. En Historia mínima ilustrada. La educación en México, (coord.) Dorothy Tanck de Estrada. México: El Colegio de México, 2011, 189-225.

Márquez Montiel, Joaquín. Hombres célebres de Chihuahua. 2a. Chihuahua: Gobierno del Estado de Chihuahua, 2004.

Pérez Piñón, Francisco Alberto. Contribución educacional de la Escuela Normal del Estado de Chihuahua en el período de la Revolución Mexicana. Chihuahua: Sindicato del Personal Académico de la UACH / Universidad Autónoma de Chihuahua, 2007. 
Pérez Piñón, Francisco Alberto, Guillermo Hernández Orozco, y Jesús Adolfo Trujillo Holguín. "«Aprender a leer y escribir en Chihuahua: siglo XIX»”. IE Revista de Investigación Educativa de la REDIECH (Red de Investigadores Educativos Chihuahua AC), no 9 (octubre 2014): 35-42.

Serrano Álvarez, Pablo. Porfirio Díaz y el Porfiriato. Cronología 18301915. México: Instituto Nacional de Estudios Históricos de las Revoluciones de México, 2012.

Trujillo Holguín, Jesús Adolfo. La educación socialista en Chihuahua 19341940, una mirada desde la Escuela Normal del Estado. Chihuahua: Universidad Autónoma de Chihuahua/Sindicato del Personal Académico de la UACH, 2015.

Trujillo Holguín, Jesús Adolfo. Sembradores: La Normal del Estado en la historia educativa de Chihuahua. Chihuahua: Secretaría de Educación y Cultura, 2005.

Vargas Valdés, Jesús. La utopía de la educación porfiriana en Chihuahua y los afanes para fundar una escuela de maestros (1890-1910). Vol. II, El Chihuahua horizontes de su historia y su cultura, (coord.) Jesús Vargas Valdés, México: Grupo Editorial Milenio, 2010, 104-121.

Vargas Valdés, Jesús. Mariano Irigoyen Escontrías, una vida por la educación. Chihuahua: Gobierno del Estado de Chihuahua, 2002.

Para citar este artículo: Trujillo Holguín, Jesús Adolfo; Pérez Piñón, Francisco Alberto y Hernández Orozco, Guillermo. "Del esplendor porfirista al caos revolucionario. La enseñanza primaria en el Estado de Chihuahua, México, en el período 1892 a 1911”, Historia Caribe Vol. XIII No. 32 (Enero-Junio 2018): 143-169. DOI: http://dx.doi.org/10.15648/ hc.32.2018.6 